ARTICLE

DOI: $10.1038 / \mathrm{s} 41467-017-01024-2$

\title{
PKC $\varepsilon$ phosphorylates MIIP and promotes colorectal cancer metastasis through inhibition of RelA deacetylation
}

Tao Chen ${ }^{1}$, Jingjie $\mathrm{Li}^{2}$, Meidong $\mathrm{Xu}^{1}$, Qin Zhao ${ }^{2}$, Yingyong Hou ${ }^{3}$, Liqing Yao ${ }^{1}$, Yunshi Zhong ${ }^{1}$, Ping-Chieh Chou ${ }^{4}$, Wei Zhang ${ }^{4}$, Pinghong Zhou ${ }^{1} \&$ Yuhui Jiang (1) ${ }^{2}$

EGFR signaling is implicated in NF- $\mathrm{BB}$ activation. However, the concrete mechanisms by which the core transducer of NF- $\mathrm{BB}$ signaling pathway, RelA/p65 is regulated under EGFR activation remains to be further clarified. Here, we show that EGF stimulation induces PKC $\varepsilon^{-}$ dependent phosphorylation of migration and invasion inhibitory protein (MIIP) at Ser303; this phosphorylation promotes the interaction between MIIP and RelA in the nucleus, by which MIIP prevents histone deacetylase 6 (HDAC6)-mediated RelA deacetylation, thus enhances transcriptional activity of RelA and facilitates tumor metastasis. Meanwhile PP1, which functions as a phosphatase, is found to mediate MIIP-S303 dephosphorylation and its expression level inversely correlates with metastatic capability of tumor cells. Moreover, clinical analyses indicate the level of MIIP-S303 phosphorylation correlates with colorectal cancer (CRC) metastasis and prognosis. These findings uncover an unidentified mechanism underlying the precise regulation of $\mathrm{NF}-\kappa \mathrm{B}$ by $\mathrm{EGF}$, and highlight the critical role of nuclear MIIP in tumor metastasis.

\footnotetext{
${ }^{1}$ Endoscopy Center and Endoscopy Research Institute, Zhongshan Hospital, Fudan University, Shanghai 200032, China. ${ }^{2}$ The Institute of Cell Metabolism Shanghai Key Laboratory of Pancreatic disease, Shanghai General Hospital, School of Medicine, Shanghai Jiaotong University, Shanghai 201620, China. ${ }^{3}$ Department of Pathology, Zhongshan Hospital, Fudan University, Shanghai 200032, China. ${ }^{4}$ Department of Cancer Biology, Comprehensive Cancer Center of Wake Forest Baptist Medical Center, Winston-Salem, NC 27157, USA. Tao Chen and Jingjie Li contributed equally to this work. Correspondence and requests for materials should be addressed to W.Z. (email: wezhang@wakehealth.edu) or to P.Z. (email: zhou.pinghong@zs-hospital.sh.cn) or to Y.J. (email: yhjiang@shsmu.edu.cn)
} 
$\mathrm{N}$ F- $\mathrm{BB}$ signaling pathway is physiologically linked to inflammatory process and is basically involved in the regulation of cellular growth and survival ${ }^{1,2}$. Its dysregulation has been implicated in the initiation and progression of tumor

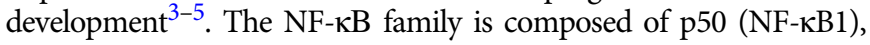
p52 (NF-кB2), p65 (RelA), c-Rel, and RelB and functions in the form of heterodimeric and homodimeric complexes ${ }^{6}$. In general, $\mathrm{NF}-\kappa \mathrm{B}$ is able to be activated via two distinct pathways under various stimuli such like cytokines, growth factors, and oncoproteins. In the canonical pathway, under basal conditions cytoplasmic NF- $\kappa B$ binds to their inhibitors IKB. Stimulation of the cell triggers TGF $\beta$ activated kinase 1 (TAK1)-dependent activation of IкB kinase (IKK) complex (IKK $\alpha$, IKK $\beta$, and IKK $\gamma / \mathrm{NEMO})^{7}$. The IKK activation phosphorylates I $\mathrm{KB}$ and promotes its proteasomal degradation, which subsequently leads to nuclear translocation of NF- $\mathrm{KB}$; thereby facilitates the gene transcription of NF- $\kappa \mathrm{B}$-targeted genes ${ }^{7}$. On the other hand, NF- $\mathrm{\kappa B}$ activation can be triggered in a non-canonical manner in which NF- $\kappa B$ is cleaved by IKK $\alpha$, through a process dependent NF- $\kappa \mathrm{B}$-inducing kinase (NIK) but IKK $\beta$ and IKK $\gamma$.

The regulation of the $\mathrm{NF}-\kappa \mathrm{B}$ signal usually becomes more complicated in cross-talking with other cellular signals, as a result its consequent effect is determined in a diverse manner. The cooperative effect between EGFR and NF- $\kappa \mathrm{B}$ pathways is importantly implicated in tumourigenesis ${ }^{8}$, among which $\mathrm{PKC} \varepsilon$ signaling has been known involved in EGF-induced NF- $\mathrm{KB}$ activation by its direct phosphorylation on IKK $\beta$ that eventually leads to RelA activation ${ }^{9,10}$. As the core signaling transducer of NF- $\mathrm{BB}$ pathway, RelA is regulated flexibly with respect to the status of its translational modification including phosphorylation and acetylation ${ }^{11}$. Acetylation in distinct lysine residues affects NF- $\kappa B$ activity differently. For instance, lysine 221 acetylation of RelA selectively enhances its DNA binding while lysine 310 acetylation facilitates its full transcriptional activity independent of regulation of DNA binding or I- $\kappa$ Balpha binding ${ }^{12}$. In turn, acetylated RelA is deacetylated by histone deacetylase 3 (HDAC3). Deacetylation of lysine 221 promotes high-affinity binding of RelA. In this layer, to further study the mechanisms underlying the regulation of RelA activity in the context of $\mathrm{EGF} / \mathrm{PKC} \varepsilon / \mathrm{NF}-\kappa \mathrm{B}$ pathway will be helpful for better understanding the relevant physiological impact.

The migration and invasion inhibitory protein (MIIP) is recognized as a repressor in the regulation of cell growth and invasion $^{13,14}$. Previous studies indicated MIIP antagonizes insulinlike growth factor binding protein 2 (IGFBP-2)-mediated invasion in glioma cell ${ }^{15}$, and is able to inhibit the enzymatic activity of Histone deacetylase 6 (HDAC6) against $\alpha$-tubulin acetylation that is related to reduction of cell migration ${ }^{16}$. In addition, MIIP was found to promote EGFR protein degradation and exert the negative effect on proliferation in lung cancer cells ${ }^{17}$. Of note, a recent study suggests nuclear HDAC6 inhibits invasion by suppressing NF- $\kappa \mathrm{B} /$ MMP2 signaling ${ }^{18}$. Given on the implication of functional relationship between MIIP and HDAC6, the potential regulatory effect of MIIP on HDAC6 in the nucleus is worthy of investigation to uncover the precise role of MIIP during cell migration and invasion.

Here, we show that activation of EGFR in human cancer cells results in PKCE-dependent MIIP phosphorylation and its interaction with RelA in the nucleus. Intriguingly, phosphorylated MIIP protects deacetylation of RelA from HDAC6, thereby ensures EGFR-stimulated RelA transcriptional activity and potentiates tumor metastasis. Furthermore, PP1 is found to mediate MIIP-S303 dephosphorylation and its downregulation is responsible for the metastatic capability of tumor cells.

\section{Results}

EGF induces the Interaction between MIIP and RelA. Based on the vital role of NF- $\mathrm{\kappa B}$ signals and MIIP in tumor metastasis, we first examined whether MIIP is involved in the EGF-induced NF$\kappa \mathrm{B}$ activation. Nuclear fraction followed by an immunoprecipitation analysis in HCT116 cells indicated EGF stimulation resulted in a dramatic increase of the interaction between endogenous MIIP and RelA in the nucleus (Fig. 1a and Supplementary Fig. 1a). To investigate the potential effects of nuclear MIIP on $\mathrm{NF}-\kappa \mathrm{B}$ activity in the level of translational modification, we expressed MIIP shRNAs in HCT116 cells (Supplementary Fig. 1b). Intriguingly, MIIP depletion resulted by according MIIP shRNAs efficiently abrogated EGF-induced RelA acetylation at K310 but not phosphorylation at S536 (Fig. 1b and Supplementary Fig. 1c), both of which are related to RelA transcriptional activity in nucleus ${ }^{11}$. We next examined whether MIIP directly regulates tumor cell invasion and the relevant genes transcription. Consistently with previous reports ${ }^{16}$, transwell analysis of HCT116 cells showed MIIP depletion increased the basal level of cell invasion. However, either EGF-enhanced tumor cell invasion (Fig. 1c) or EGF-induced transcription of MMP2 and Twist (Supplementary Fig. 1d) was compromised by MIIP depletion. These effects were recapitulated in cells with overexpression of acetylation resistant RelA K310R mutant to more profound extents but not its wild type (WT) counterpart (Fig. 1d, e), suggesting the importance of RelA activity in EGF-induced tumor cell invasion. In addition, ChIP analysis showed EGF treatment dramatically increased accumulation of MIIP and RelA AcK310 at promoter regions of MMP2 and Twist genes, which were disrupted by MIIP depletion (Fig. 1f).

PKC $\varepsilon$ is reported to be responsible for RelA activation upon EGF stimulation ${ }^{9,10}$. In agreement, PKC inhibitor Bis-I treatment led to impairment of RelA acetylation induced by EGF (Supplementary Fig. 1e). In the meantime, we stably expressed Flag-MIIP in HCT116 cells and found the binding of MIIP to RelA induced by EGF treatment was blocked with PKC inhibition but not treatment with MEK/ERK inhibitor U0126 (Fig. 1g). These results suggest EGF-induced interaction between RelA and MIIP would be attributed to augmented EGFR-PKCE signaling, which thus drive us to further explore the underlying mechanism. As shown in the left panel of Fig. 1h, purified recombinant MIIP protein were incubated with nuclear extracts from HCT116 cells, and GST pull down analysis indicates MIIP has no binding to nuclear RelA regardless of EGF treatment. However, another parallel GST pull down assay showed EGF stimulation significantly promoted the association of nuclear MIIP with purified recombinant RelA protein (Fig. 1h, the right panel). These data suggest the EGF-induced interaction between MIIP and RelA is regulated in a manner dependent on altered status of MIIP but not RelA.

PKCe phosphorylates MIIP and promotes MIIP-RelA interaction. We further investigated the key regulatory signals required for the association between MIIP and RelA under EGFR activation. Co-immuoprecipitation analysis showed EGF-induced interaction between MIIP and RelA was abolished in the precipitates incubated with calf intestinal phosphatase (CIP) (Fig. 2a), revealing the interaction is phosphorylation dependent. Along with the results from the GST pull down assay (Fig. 1h), this finding raised the possibility that cellular MIIP might undergo translational modification through EGFR-PKCe pathway. An in vitro protein phosphorylation assay indicated that purified, activative PKC $\varepsilon$ phosphorylated purified recombinant MIIP and the phosphorylation was detected by immunoblotting analyses only with an anti-phospho-Serine antibody (Fig. 2b), as demonstrated by autoradiography and immunoblotting analysis. In addition, Scansite analysis also showed Ser303 and Thr158 on MIIP are potential PKCe phosphorylated residues, and the 
autoradiography analysis further showed the mutation of evolutionarily conserved Ser 303 (Fig. 2c, left panel) largely abolished PKCE-mediated MIIP phosphorylation, which is also revealed in an immunoblotting analysis with a specific MIIP-S303 phosphorylation antibody (Fig. $2 c$, the right panel).

To determine whether PKCe-mediated MIIP-S303 phosphorylation is required for EGF-induced MIIP-RelA interaction, we depleted endogenous MIIP in HCT116 cells and reconstituted the expression of RNAi resistant WT rMIIP and rMIIP S303A (Supplementary Fig. 2a), and the co-immunoprecipitation analysis were performed. In contrast to its Flag-tagged WT counterpart, MIIP S303A mutant which is resistant to MIIP-S303 phosphorylation, largely lost its interaction with RelA under EGF stimulation (Fig. 2d). Meanwhile, we found rMIIP S303A, but not WT rMIIP resulted in compromised enhancement of RelA AcK310 levels induced by EGF stimulation (Fig. 2d). In addition, the effects of MIIP S303A on MIIP-RelA interaction and RelA AcK310 were recapitulated by PKC $\varepsilon$ depletion (Supplementary Fig. 2b). In accordance with these results, both EGF-induced recruitment of MIIP (Fig. 2e) and accumulated RelA AcK310 (Fig. 2f) at promoter regions of MMP2 and Twist genes were conspicuously blocked in HCT116 cells expressed with rMIIP


C
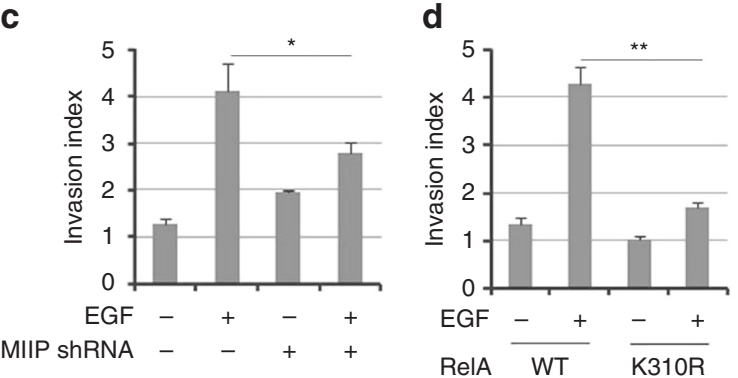

f
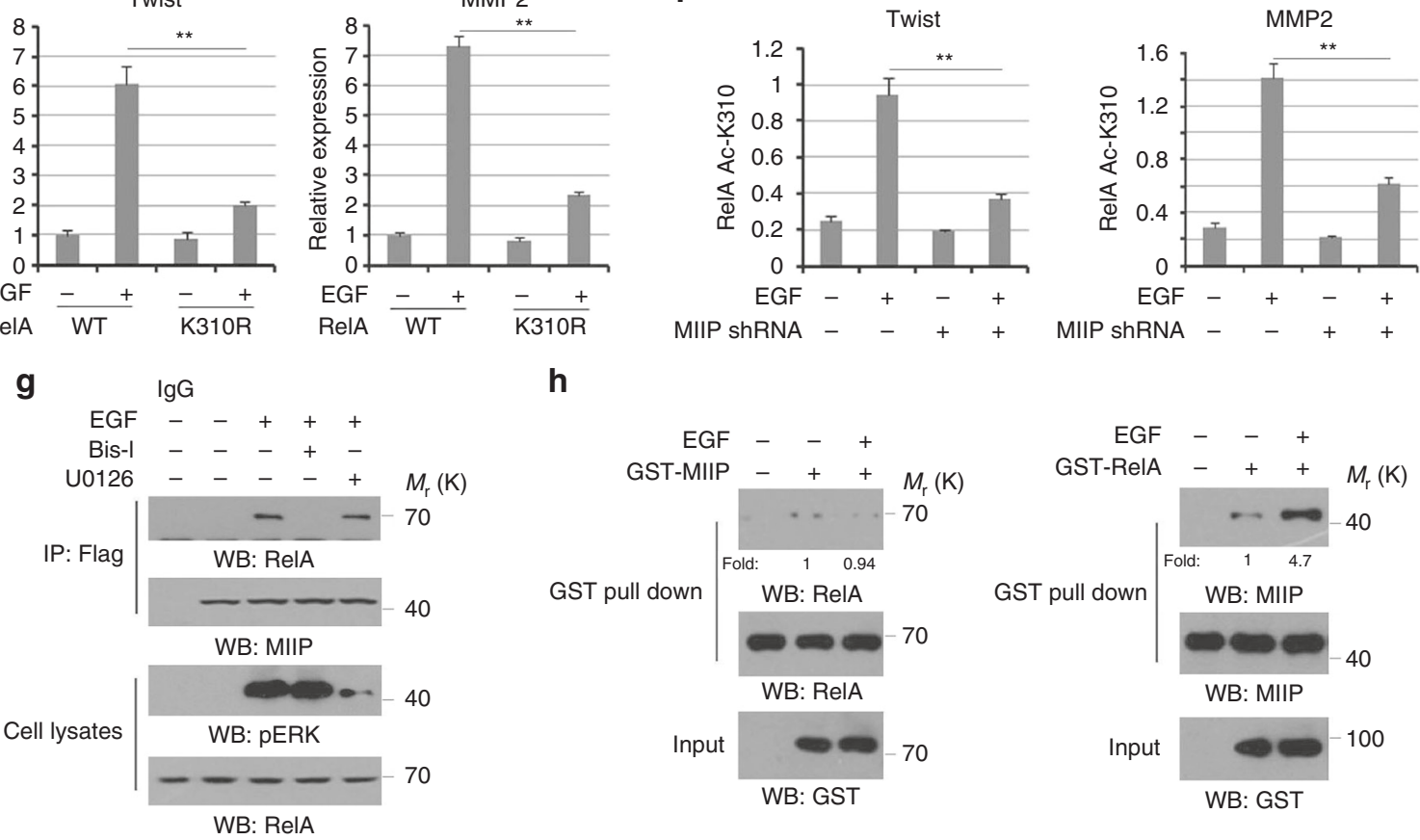

h
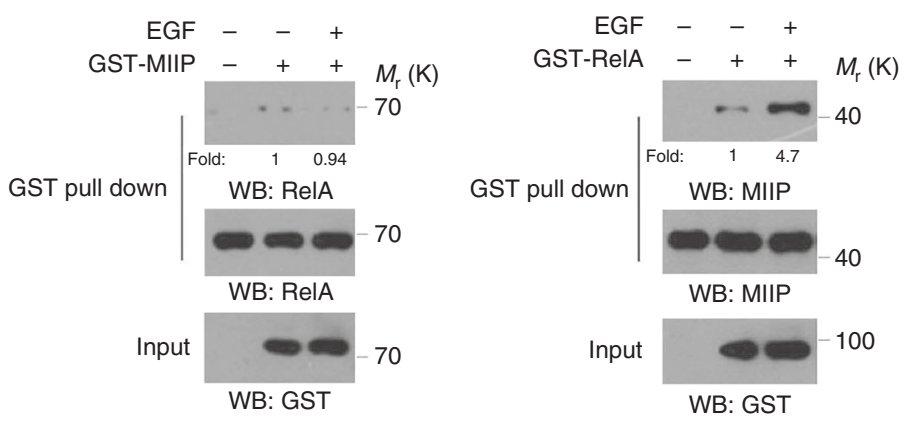

Fig. 1 EGF induced the interaction between MIIP and RelA. a HCT116 cells were treated with or without EGF. Cellular nucleus-extracts subjected to immunoprecipitation with an anti-MIIP antibody. $\mathbf{b}$ HCT116 cells transfected with or without plasmid for expressing the indicated MIIP shRNA were treated with or without EGF for indicated periods of time. c, d HCT116 cells transfected with or without plasmid for expressing MIIP shRNA (c), and HCT116 cells expressed with wild type (WT) RelA and RelA K310R (d) were treated with or without EGF $(100 \mathrm{ng} / \mathrm{ml})$. Cell invasion assays were performed. e HCT116 cells expressed with WT RelA and RelA K310R were treated with or without EGF $(100 \mathrm{ng} / \mathrm{ml})$ for $10 \mathrm{~h}$. Relative mRNA levels were analyzed by q-PCR. $\mathbf{f}$ HCT116 cells transfected with or without plasmid for expressing MIIP shRNA were treated with or without EGF (100 ng/ml) for $10 \mathrm{~h}$. ChIP analyses with an anti-RelA Ac-K310 antibody were performed. The primers covering RelA binding site of MMP2 gene promoter region were used for the q-PCR. The $Y$ axis shows the value normalized to the input. $\mathbf{g}$ HCT116 cells were pretreated with Bis-I $(2 \mu \mathrm{M}), \mathrm{U} 0126(20 \mu \mathrm{M})$ for $1 \mathrm{~h}$, prior to EGF treatment (100 ng/ml) for $30 \mathrm{~min}$. Cellular extracts subjected to immunoprecipitation with an anti-Flag antibody. h Purified GST-MIIP protein was mixed with mitotic extracts from HCT116 cells treated with EGF (100 ng/ml) for 30 min. GST pull down analyses were performed (left panel). Purified GST-RelA protein was mixed with mitotic extracts from HCT116 cells treated with EGF $(100 \mathrm{ng} / \mathrm{ml})$ for 30 min (right panel). In $\mathbf{a}, \mathbf{b}, \mathbf{g}$, $\mathbf{h}$, immunoblotting analyses were performed using the indicated antibodies and data represent one out of three experiments. In c-f, the values are presented as mean \pm s.e.m. ( $n=3$ independent experiments), * represents $P<0.05$ and ${ }^{\star *}$ represents $P<0.01$ (Student's $t$-test) between the indicated groups 
a



b $\mathrm{PKC}+\ldots+$ C

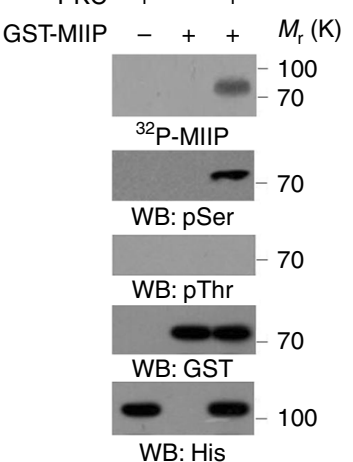

303

Human RYHIHRRKŠFDASDTLA

Chimpanzee RYHIHRRKSFDASDTLA

Mouse QYRIHRRKSFDASDTLA

Rat QYRIHRRKSFDASDTFA

Chicken KYKAHCRKSFEPADSLA

Zebrafish KYKAHRRSSFDPSDSLG

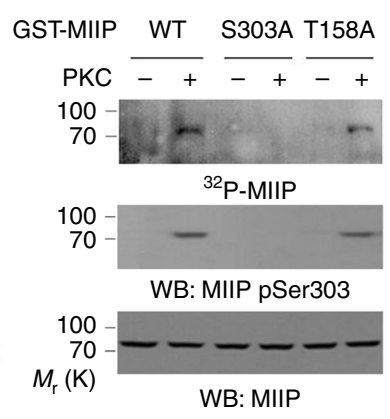

f

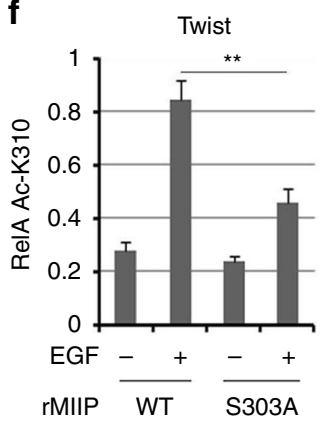

d



g



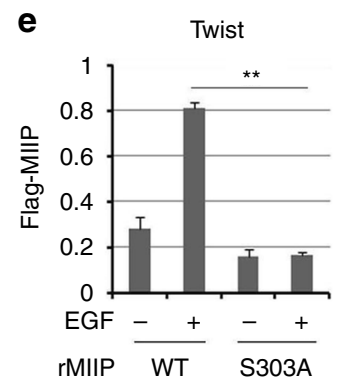
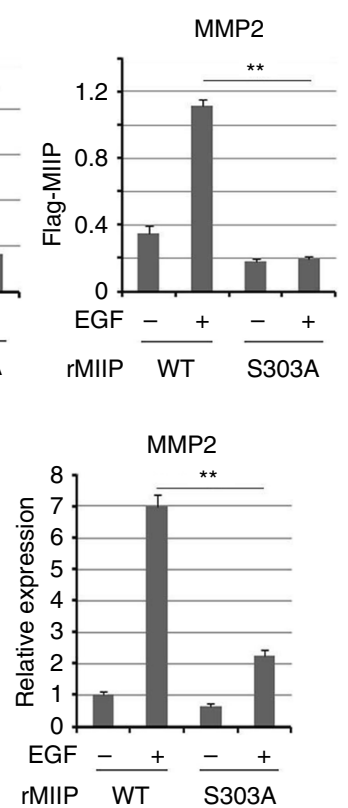

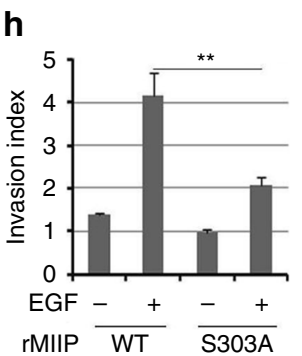

Fig. 2 PKC $\varepsilon$ phosphorylated MIIP and promoted MIIP-RelA interaction. a HCT116 cells were treated with or without EGF. Cellular extracts subjected to immunoprecipitation with an anti-Flag antibody. The immunoprecipitates were treated with CIP (10 units), followed by immunoblotting analysis. b, c In vitro phosphorylation analyses were performed by mixing the purified active PKC $\varepsilon$ with the indicated purified GST-MIIP proteins in the presence of $[\gamma$-32P]ATP. Ser303 of MIIP is evolutionarily conserved in the indicated species (c, left panel). d HCT116 cells expressed with WT MIIP or MIIP S303A were treated with or without EGF for 30 min. e HCT116 cells expressed with WT MIIP or MIIP S303A were treated with or without EGF (100 ng/ml) for $10 \mathrm{~h}$. f HCT116 cells with depletion of MIIP, and reconstituted expression of WT rMIIP or rMIIP S303A were treated with or without EGF (100 ng/ml) for $10 \mathrm{~h}$.

g HCT116 cells with depletion of MIIP, and reconstituted expression of WT rMIIP or rMIIP S303A were treated with or without EGF for $10 \mathrm{~h}$. Relative mRNA levels were analyzed by q-PCR. h HCT116 cells with depletion of MIIP, and reconstituted expression of WT rMIIP or rMIIP S303A were treated with or without EGF (100 ng/ml). Cell invasion assays were performed. In e, f, ChIP analyses with indicated antibodies were performed. The primers covering RelA binding site of MMP2 gene promoter region were used for the q-PCR. The $Y$ axis shows the value normalized to the input. In a-d, immunoblotting analyses were performed using the indicated antibodies. In $\mathbf{e}-\mathbf{h}$, the values are presented as mean \pm s.e.m. $(n=3$ independent experiments), ** represents $P<0.01$ (Student's $t$-test) between the indicated groups

S303A compared with its WT counterpart. Examination of MMP2 and Twist mRNA levels also showed EGF-induced downstream transcription was compromised by reconstituted expression of rMIIP S303A in HCT116 cells (Fig. 2g), which mirrored (Fig. 1e) but did not aggravate (Supplementary Fig. 2c) the effects from RelA K310R expression. Furthermore, transwell analysis showed rMIIP S303A expression significantly inhibited EGF-induced tumor cell invasion (Fig. 2h), and no additional effects on impaired invasive ability were observed when rMIIP S303A mutant co-expressed with RelA K310R in HCT116 cells compared with its WT counterpart (Supplementary Fig. 2d). Collectively, these results suggest PKC 8 -mediated MIIP-S303 phosphorylation facilitates the interaction between MIIP and RelA, which is required for EGF-induced tumor cell invasion.
PP1 mediates MIIP dephosphorylation. To further evaluate the positive effects of PKC 8 -mediated MIIP phosphorylation on tumor cell invasion, we examined MIIP-S303 phosphorylation levels in multiple colorectal cancer (CRC) cell lines with distinct invasive capability. Apparently, immunoblotting analysis showed the EGF stimulated stronger MIIP-S303 phosphorylation in CRC cells (Supplementary Fig. 3a) which displayed the higher tendency of invasion (Supplementary Fig. 3b), implying there would be certain cellular component that counteracts PKCE-mediated MIIP phosphoryaltion and restricts invasive ability of tumor cells. The cellular amount of eukaryotic protein phosphorylation is reversibly regulated through phsophatase-mediated dephosphorylation. Phosphoprotein phosphatases (PPP) superfamily includes PP1, PP2A, and is known to participate in diverse 

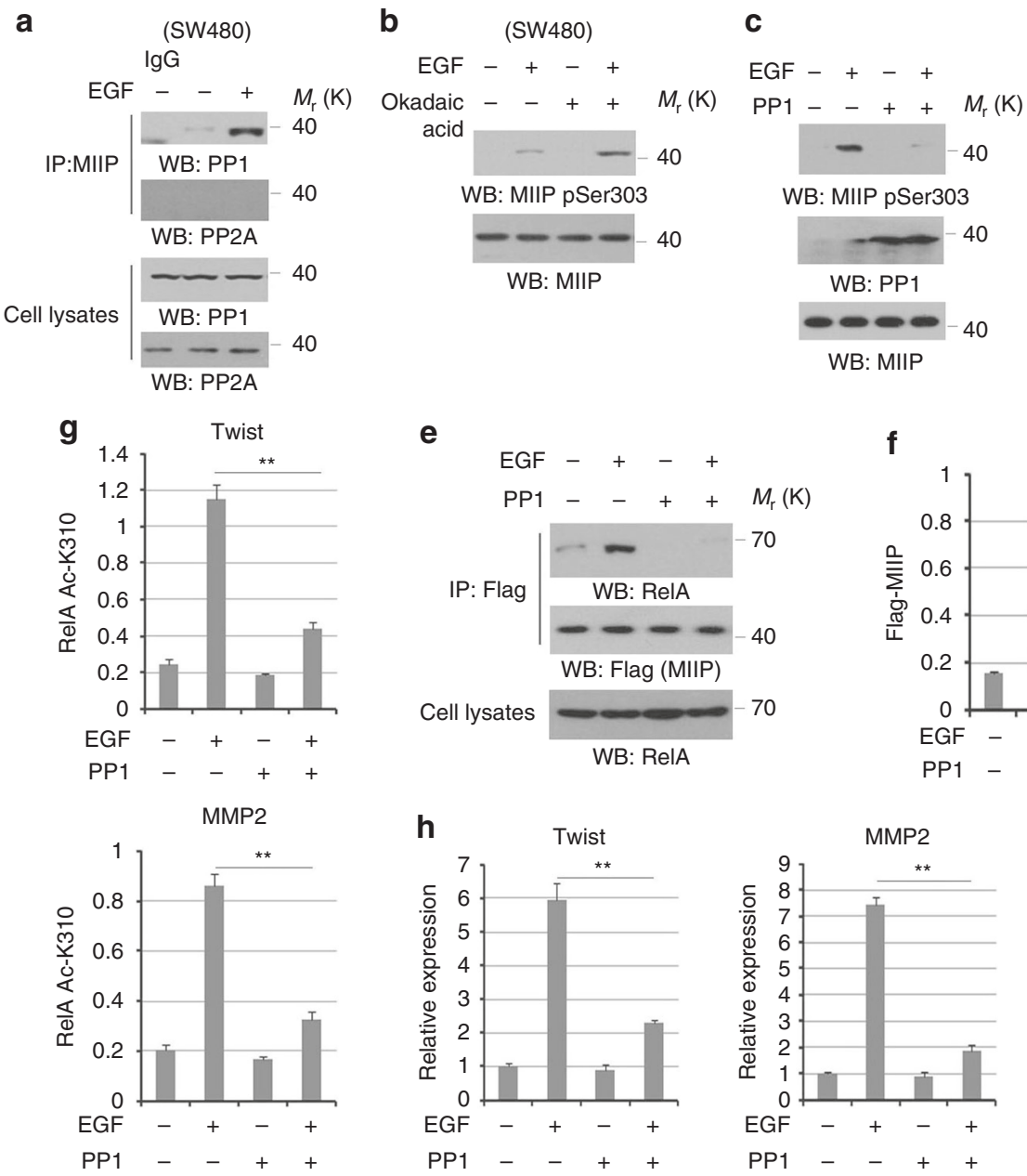

e
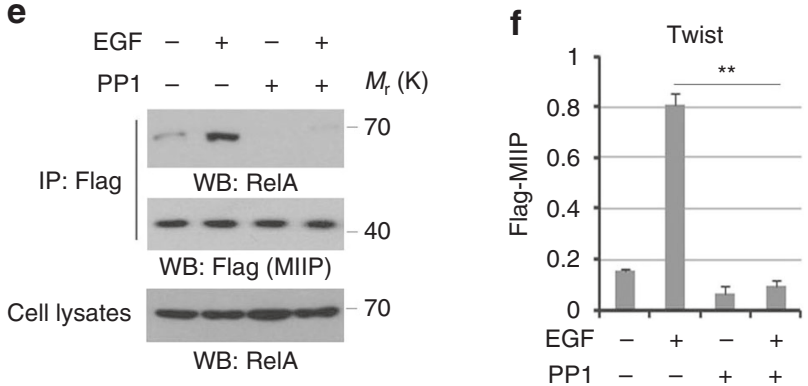
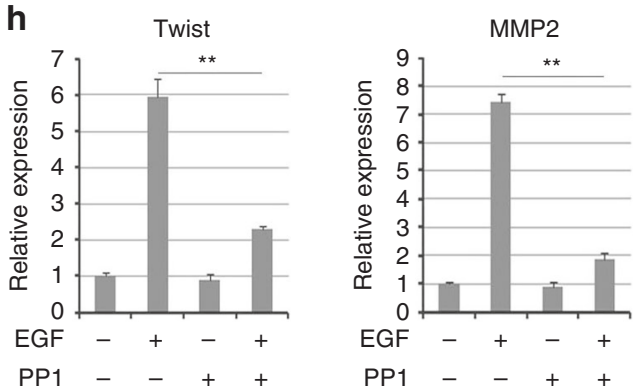
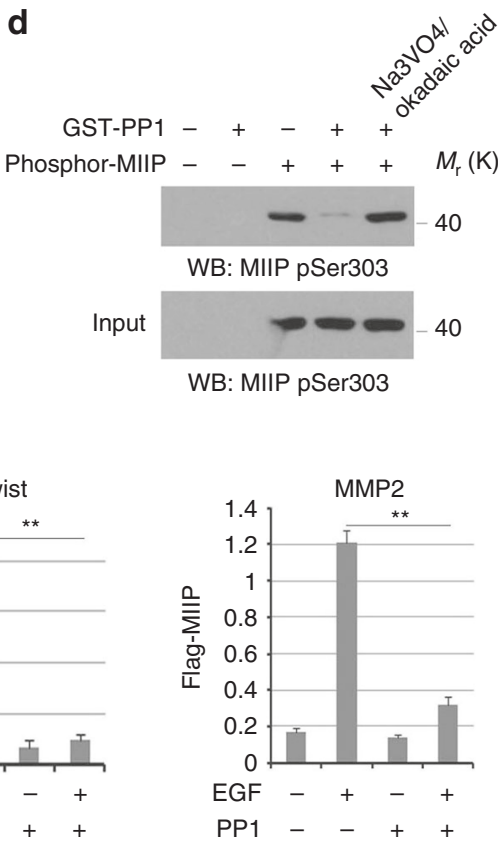

i

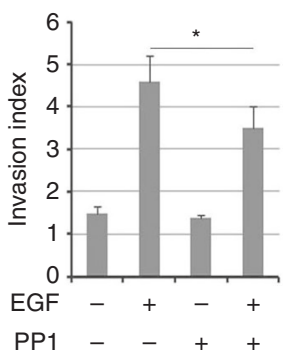

Fig. 3 PP1 mediates MIIP dephosphorylation. a SW480 cells were treated with or without EGF. Cellular extracts subjected to immunoprecipitation with an anti-MIIP. b SW480 cells pretreated with Okadaic acid (a PP1 and PP2A inhibitor) ( $30 \mathrm{nM}$ ) were stimulated with or without EGF (100 ng/ml) for 30 min. c HCT116 cells transfected with or without plasmid for expressing PP1 were treated with or without EGF (100 ng/ml) for $30 \mathrm{~min}$. d In vitro dephosphorylation analyses were performed by mixing the purified active PP1 with PKC $\varepsilon$-phosphorylated GST-MIIP proteins in absence or presence of Na3VO4/Okadaic acid. e-h HCT116 cells expressing Flag-MIIP were transfected with or without plasmid for expressing PP1. Cells were treated with or without EGF (100 ng/ml) for $10 \mathrm{~h}$. Cellular extracts subjected to immunoprecipitation with an anti-MIIP (e). ChIP analyses were performed. The primers covering RelA binding site of Twist or MMP2 gene promoter region were used for the q-PCR (f, g). Relative mRNA levels were analyzed by q-PCR (h). (i) HCT116 cells transfected with or without plasmid for expressing PP1 was treated with or without EGF $(100 \mathrm{ng} / \mathrm{ml})$. Cell invasion assays were performed. In $\mathbf{f}$, $\mathbf{g}$, ChIP analyses with indicated antibodies were performed. The primers covering RelA binding site of MMP2 gene promoter region were used for the q-PCR. The $Y$ axis shows the value normalized to the input. In a-e, immunoblotting analyses were performed using the indicated antibodies. In a-e, immunoblotting analyses were performed using the indicated antibodies and data represent one out of three experiments. $\ln \mathbf{g}, \mathbf{f}-\mathbf{i}$, the values are presented as mean \pm s.e.m. ( $n=3$ independent experiments), * represents $P<0.05$ and ${ }^{\star *}$ represents $P<0.01$ (Student's $t$-test) between the indicated groups

biological processes ${ }^{19}$. We wondered that whether MIIP pSer303 is targeted by PP1. Interestingly, co-immuoprecipitation analysis showed the dramatic increased PP1, the catalytic subunit of PP1, but not PP2A was detected in precipitates from MIIP antibody in SW480 cells after EGF stimulation (Fig. 3a). Furthermore, we found either treatment with okadaic acid (OA), an inhibitor for both PP1 and PP2A, markedly enhanced EGF-induced MIIPS303 phosphorylation in SW480 cells (Fig. 3b), and overexpression of PP1 (Fig. 3c) robustly downregulated MIIP-S303 phosphorylation in HCT116 cells with EGF stimulation. To support these findings, the dephosphorylation assay also showed purified PP1 efficiently abolished MIIP-S303 phosphorylation of purified Flag-MIIP from HCT116 cells, which is reversed by the inhibitors Na3VO4 and OA (Fig. 3d). Thus, these data demonstrate PP1 functions as a physiological MIIP phosphatase.

The effects of PP1 activity on RelA-MIIP complexes were further demonstrated by co-immunoprecipitation and ChIP analyses showing that the interaction between RelA and MIIP (Fig. 3e), as well as the accumulation of MIIP (Fig. 3f) and RelA K310 acetylation (Fig. 3g) at promoter regions of MMP2 and Twist were disrupted in HCT116 cells with overexpression of PP1. Accordingly, transcriptions of MMP2 and Twist were also blocked by PP1 induced by EGF (Fig. 3h). However, transwell analysis showed invasion induced by EGF was attenuated by overexpression of PP1 in HCT116 cells to a lesser extent compared with that effect from MIIP S303A (Fig. 3i), suggesting the additional function of PP1 is implicated in tumor cell invasion. In contrast, PP1 depletion in SW480 cells (Supplementary Fig. 3c) promoted EGF-induced MIIP-S303 phosphorylation as well as RelA-MIIP interaction (Supplementary Fig. 3d), which is accompanied by the dramatically increased RelA K310 acetylation (Supplementary Fig. 3e) and MIIP enrichment at promoters (Supplementary Fig. 3f), and MMP2 and Twist expression levels (Supplementary Fig. 3g). 
a



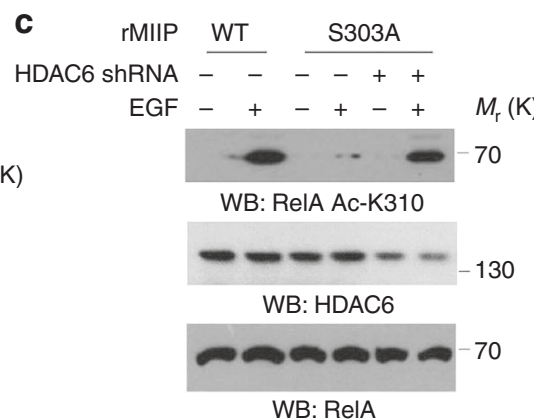

WB: RelA d

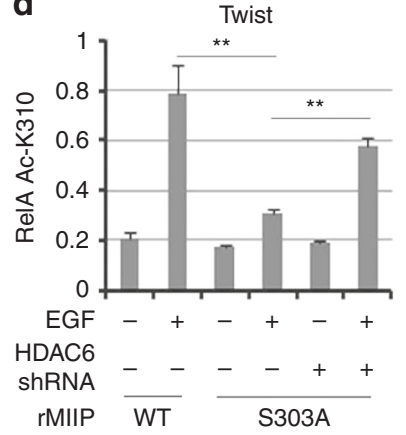

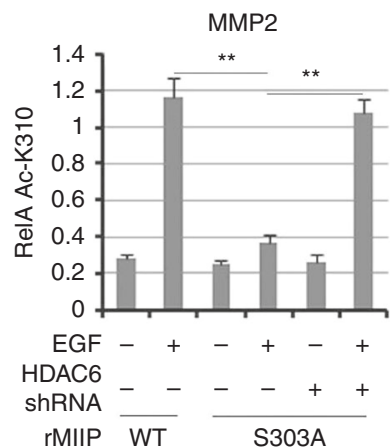

b



e

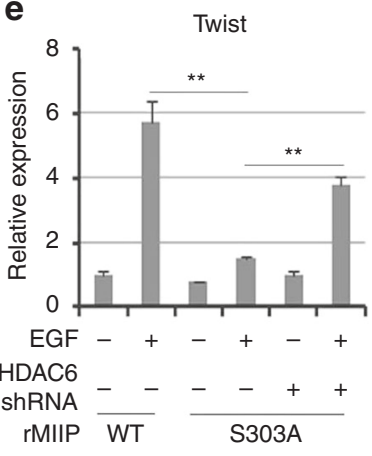

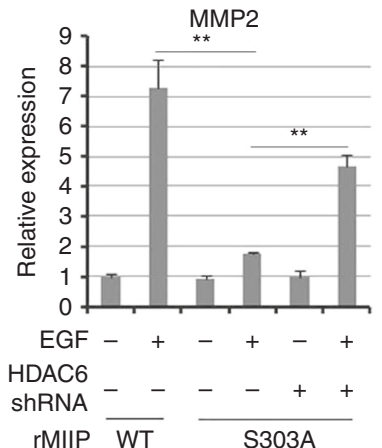

Fig. 4 MIIP prevent HDAC6-mediated RelA deacetylation. a HCT116 cells were pretreated with or without 4SC-202 (0.6 $\mu$ M), FK228(20 nM) and Nexturastat A $(5 \mathrm{nM})$ for $1 \mathrm{~h}$ prior to EGF $(100 \mathrm{ng} / \mathrm{ml})$ treatment for $30 \mathrm{~min}$. Immunoblotting analyses were performed. b HCT116 cells were pretreated with or without Bis-I for $1 \mathrm{~h}$ prior to EGF $(100 \mathrm{ng} / \mathrm{ml})$ treatment for $30 \mathrm{~min}$. Cellular extracts subjected to immunoprecipitation with an anti-Flag, followed by Flag-beads washing and a second immunoprecipitation with an anti-MIIP (lanes 1-4 from left). Cellular extracts subjected to immunoprecipitation with an anti-Flag (lanes 5-8 from left). c-e HCT116 cells expressed with WT MIIP or MIIP S303A were overexpressed with or without HDAC6 shRNA; cells were treated with or without EGF $(100 \mathrm{ng} / \mathrm{ml})$ for $10 \mathrm{~h}$. Immunoblotting analyses were performed (c). ChIP analyses with an anti-RelA Ac-K310 antibody were performed. The primers covering RelA binding site of Twist or MMP2 gene promoter region were used for the q-PCR. The $Y$ axis shows the value normalized to the input. $\mathbf{d}$ Relative mRNA levels were analyzed by q-PCR (e). In a-c, immunoblotting analyses were performed using the indicated antibodies and data represent one out of three experiments. In d, e, the values are presented as mean \pm s.e.m. ( $n=3$ independent experiments),

${ }^{\star}$ represents $P<0.01$ (Student's $t$-test) between the indicated groups

MIIP prevents HDAC6-mediated RelA deacetylation. The effect of MIIP depletion on RelA deacetylation implies MIIP might maintains RelA deacetylation instead of the initial activation (Fig. 1b). To determine this, HCT116 cells were treated with a panel of HDAC inhibitors, and we found MIIP S303A-suppressed RelA K310 acetylation induced by EGF was significantly reversed by the treatment with Nexturastat $\mathrm{A}$, a selective inhibitor against HDAC6, but not 4SC-202 and FK228, which are HDAC class I inhibitors (Fig. 4a). Consistently, MIIP is known to be able to form complex with $\mathrm{HDAC}^{16}{ }^{16}$, which is importantly involved in the regulation of RelA acetylation ${ }^{18}$. Hence, we wondered whether MIIP protects RelA acetylation through inhibition of RelA deacetylation by HDAC6. A two step-co-immunoprecipation analysis showed HDAC6 is able to interact with MIIP in precipitates from Flag-RelA in EGF-stimulated HCT116 cells (Fig. 4b, the lanes 1-4), which was abolished by treatment of PKCE inhibitor due to its inhibitory effects on MIIP-RelA interaction. In another co-immunoprecipation analysis by pulling down quantified equal amounts of Flag-RelA from HCT116 cells treated with EGF, we found RelA is associated with HDAC6 (Fig. 4b, the lanes 5-8) to a comparable level to the amount of HDAC6 binding to MIIP after washing precipitates with Flag peptides (Fig. 4b. the lanes 1-4). These data indicated there is a trimmer complex formation in the order of RelA-MIIP-HDAC6, in which MIIP might prevent the direct binding of HDAC6 to RelA and thus protect RelA from HDAC6-mediated deacetylation.
Further, we found MIIP S303A remained to interact with HDAC6 although lost the binding to RelA, and the interaction was not significantly affected by EGF (Supplementary Fig. 4a), revealing MIIP-S303 phosphorylation is only responsible for the complex formation of HDAC6-MIIP-RelA and MIIP might participate in the regulation of HDAC6 function in extra cellular events. Importantly, the downregulation of RelA K310 acetylation resulted by reconstituted expression of rMIIP S303A is blocked by shRNA-mediated depletion of HDAC6 in EGF-stimulated HCT116 cells (Fig. 4c). In accordance, ChIP analyses further showed the impaired accumulation of RelA Ac-310 at promoter regions of MMP2 and Twist resulted by rMIIP S303A was largely reversed in EGF-stimulated HCT116 cells with HDAC6 depletion (Fig. 4d). In accordance, q-PCR analyses showed HDAC6 depletion abolished the inhibitory effects of rMIIP S303A on EGF-induced transcription of MMP2 and Twist (Fig. 4e). These results revealed PKCe-phosphorylated MIIP protects RelA from HDAC6-mediated deacetylation.

KRAS is mutated in HCT116 cells ${ }^{20}$. To clarify whether the regulation of RelA by MIIP is dependent on KRAS status, endogenous MIIP was depleted and the expression of RNAi resistant WT rMIIP and rMIIP S303A were reconstituted in the colon cancer cell line $\mathrm{CaCo} 2$ cells (Supplementary Fig. 4b), which express wild type-KRAS. As shown in Supplementary Fig. 4c, EGF readily induced the interaction between RelA and WT MIIP but not MIIP S303A. Similar with that of HCT116, MIIP S303A expression which blocked promoter-enrichment of MIIP 
a

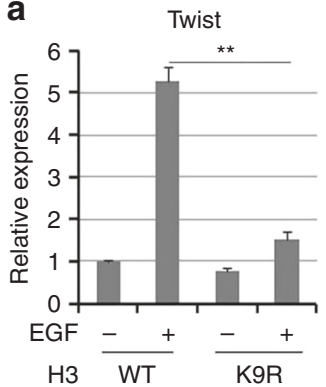

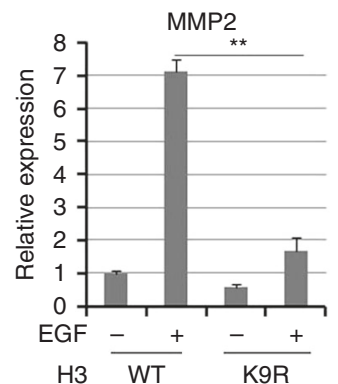

b

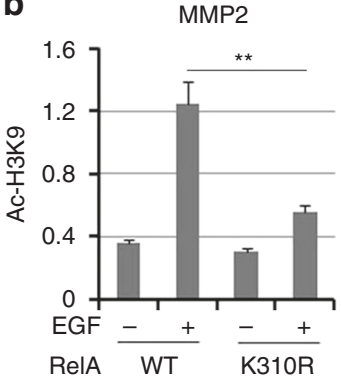

C

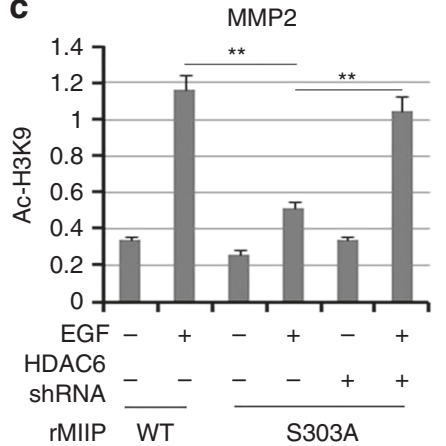

d



e

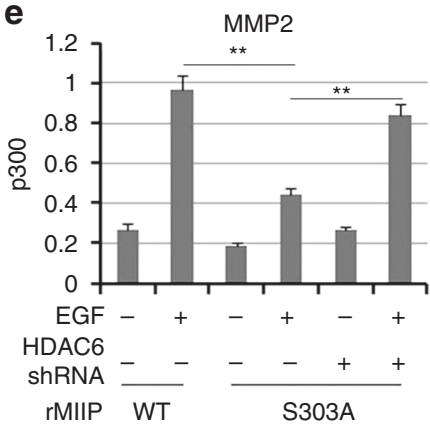

f MMP2



Fig. 5 MIIP-RelA facilitates H3-K9 acetylation at promoter region. a HCT116 cells expressed with WT H3 or H3 K9R were treated with or without EGF $(100 \mathrm{ng} / \mathrm{ml})$ for $10 \mathrm{~h}$. Relative mRNA levels were analyzed by q-PCR. b, d HCT116 cells expressed with WT RelA or RelA K310R were treated with or without EGF for $10 \mathrm{~h}(100 \mathrm{ng} / \mathrm{ml})$. c, e HCT116 cells expressed with WT MIIP or MIIP S303A were overexpressed with or without HDAC6; cells were treated with or without EGF $(100 \mathrm{ng} / \mathrm{ml})$ for $10 \mathrm{~h}$. $\mathbf{f H C T 1 1 6}$ cells transfected with or without plasmid for expressing p300 shRNA were treated with or without EGF $(100 \mathrm{ng} / \mathrm{ml})$ for $10 \mathrm{~h}$. In $\mathbf{b}-\mathbf{f}$, ChIP analyses with indicated antibodies were performed. The primers covering RelA binding site of MMP2 gene promoter region were used for the q-PCR. The $Y$ axis shows the value normalized to the input. In $\mathbf{a}-\mathbf{f}$, the values are presented as mean \pm s.e.m. ( $n=3$ independent experiments), ${ }^{\star \star}$ represents $P<0.01$ (Student's $t$-test) between the indicated groups

(Supplementary Fig. 4d) or RelA Ac-K310 in $\mathrm{CaCo} 2$ cells (Supplementary Fig. 4e), inhibited transcription of MMP2 and Twist (Supplementary Fig. 4f) and tumor cell invasion (Supplementary Fig. 4g). Meanwhile, EGF stimulation also led to the trimmer complex formation of RelA-MIIP-HDAC6 in $\mathrm{CaCo} 2$ cells (Supplementary Fig. 4h).

MIIP-RelA facilitates H3-K9 acetylation at promoter region. Increased K310 acetylation of RelA promotes its transcriptional activity for the downstream genes expression and is usually linked to the regulation of $\mathrm{H} 3$ acetylation or methylation at genes promoter region ${ }^{21-23}$. Time course analysis of various histone marks for transcription indicated accumulation of Ac- $\mathrm{H} 3 \mathrm{~K} 9$ and H3K4me3 but not Ac-H3K18 or H3K36Me2 at the MMP2 promoter region were notably increased in HCT116 cells with EGF stimulation (Supplementary Fig. 5a). Subsequently, HCT116 cells were overexpressed with WT H3 or H3 K9R mutant, in which the lysine was mutated into arginine (Supplementary Fig. 5b). ChIP analysis showed EGF stimulated a dramatic increase of $\mathrm{H} 3-\mathrm{K} 9$ acetylation at MMP2 promoter region, which was abolished by H3 K9R (Supplementary Fig. 5c). In the meantime, q-PCR analyses showed EGF-induced upregulation of Twist and MMP2 were tremendously inhibited by H3 K9R expression (Fig. 5a). We next examined whether MIIP-RelA interaction at MMP2 promoter region is involved in the regulation of histone $\mathrm{H} 3$ acetylation. As a result, EGF-induced H3-K9 acetylation at MMP2 promoter was blocked either by RelA K310R expression (Fig. 5b), or rMIIP S303A (Fig. 5c), compared with their WT counterparts. Additionally, we found rMIIP S303A induced downregulation of histone $\mathrm{H} 3-\mathrm{K} 9$ acetylation at MMP2 promoter was inhibited in HCT116 cells with HDAC6 depletion (Fig. 5c). In addition,
H3K9R overexpression largely blocked EGF-induced H3K4me3 at the MMP2 promoter region (Supplementary Fig. 5d), suggesting the prerequisite role of Ac-H3K9 for $\mathrm{H} 3 \mathrm{~K} 4 \mathrm{me} 3$ induction. Accordingly, overexpression of Rel K310R or reconstituted expression of rMIIP S303A, which inhibited promoter-associated Ac-H3K9 (Fig. 5b, c), led to decreased H3K4me3 enrichment at promoter (Supplementary Fig. 5e, f). ChIP analysis further indicated the negative effects of rMIIP S303A on H3K4me3 was reversed by HDAC6 depletion (Supplementary Fig. 5f). All together, these data suggest $\mathrm{Ac}-\mathrm{H} 3 \mathrm{~K} 9$ and $\mathrm{H} 3 \mathrm{~K} 4 \mathrm{me} 3$ are induced by EGF in a sequential manner and MIIP-mediated maintenance of RelA acetylation was critical for the subsequent accumulation of $\mathrm{H} 3-\mathrm{K} 9$ acetylation at promoter region.

To further investigate the mechanism underlying MIIP-RelAregulated histone $\mathrm{H} 3$ acetylation, we performed ChIP analyses with antibodies against p300, a well-known acetyltransferase responsible for H3-K9 acetylation ${ }^{24,} 25$. Consequently, EGF treatment promoted p300 accumulation at MMP2 promoter in HCT116 cells, which was inhibited by overexpression of RelA K310R (Fig. 5d) or MIIP S303A (Fig. 5e), compared with their WT counterparts. Consistent with its effects on H3 lysine9 acetylation, HDAC6 depletion restored p300 accumulation at MMP2 promoter in HCT116 cells with expression of MIIP S303A (Fig. 5e). Importantly, depletion of p300 largely abolished EGFinduced $\mathrm{H} 3-\mathrm{K} 9$ acetylation at MMP2 promoter region in HCT116 cells (Fig. 5f). These results suggest MIIP-RelA interaction is required for the $\mathrm{H} 3-\mathrm{K} 9$ acetylation mediated by p300 at promoter region.

MIIP-S303 phosphorylation is required for tumor metastasis. To determine whether MIIP-S303 phosphorylation promotes 
a



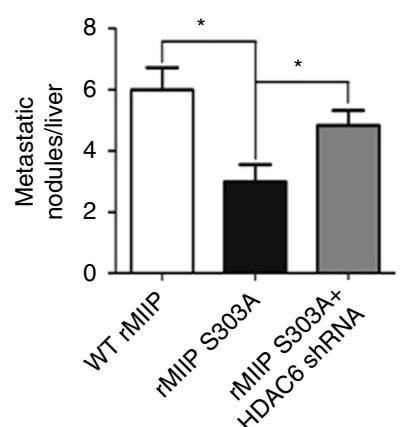



C

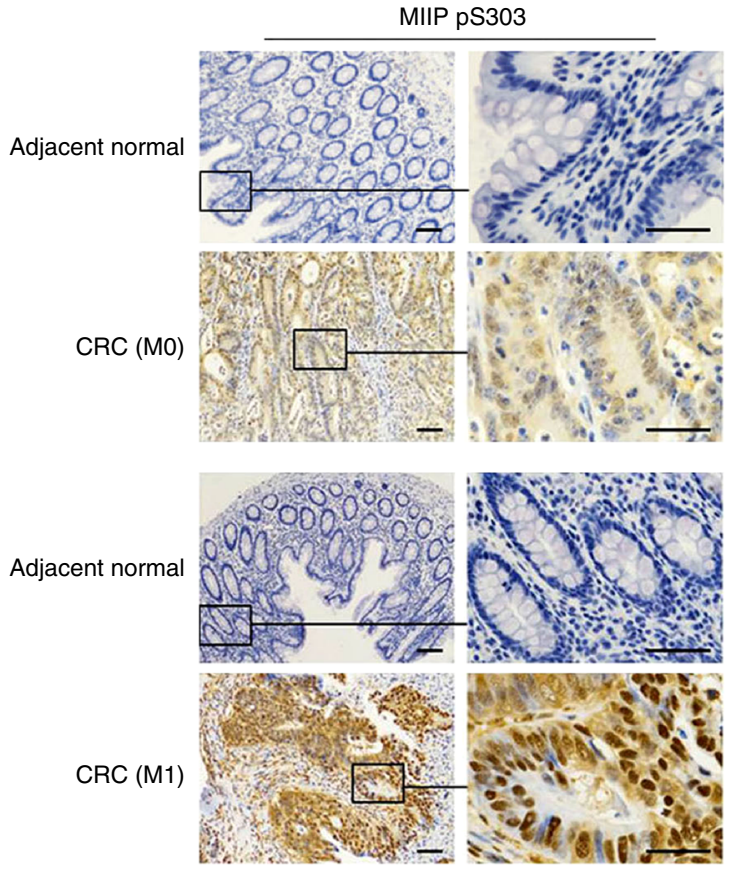

d

$$
\begin{aligned}
& \text { - Tumour } \\
& \text { - Normal }
\end{aligned}
$$

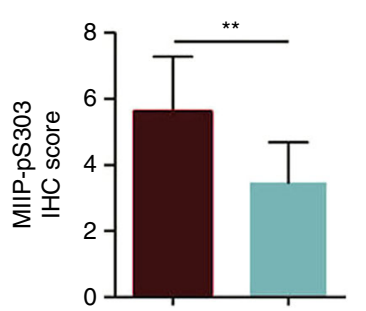

e
- High MIIP pS303

Low MIIP pS303
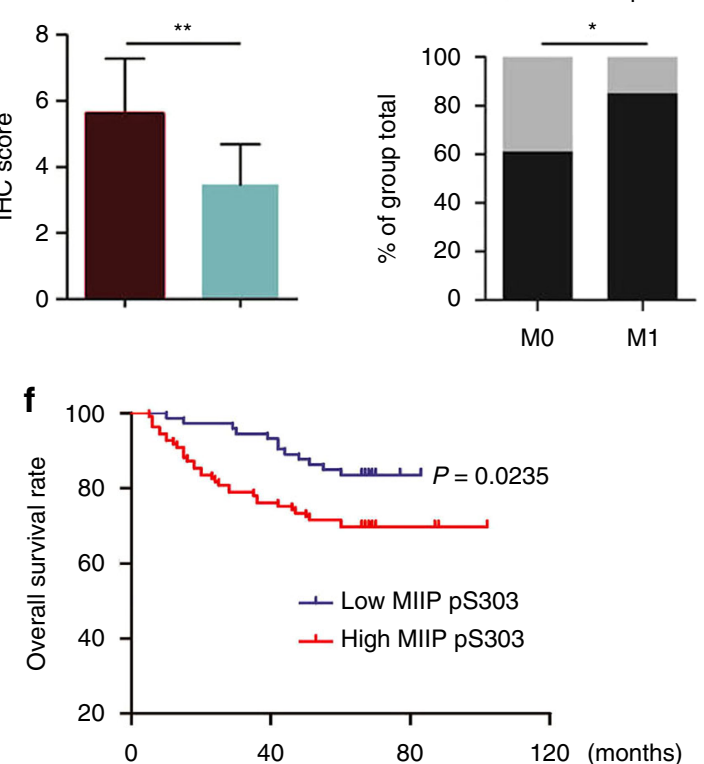

Fig. 6 MIIP-S303 phosphorylation is required for tumor metastasis and is related to poor prognosis in human colorectal cancer. a SW620 cells with reconstituted expression of WT rMIIP, rMIIP S303A and rMIIP S303A/HDAC6 shRNA were intraspleenically injected into the athymic nude mice. Representative tumor xenografts were shown (left panel). The number of visible metastatic lesions in the liver was measured. Data represent the means \pm s.e.m. $(n=8$, right panel), * represents $P<0.05$ (Student's t-test). b Lysates obtained from the pool of tumor tissues $(n=8)$ developed from SW620 cells (as indicated in Fig. 6a) were subjected to immunoblotting analyses using the indicated antibodies. c Immunohistochemical staining with anti-MIIP pS303 was performed on 182 human colorectal cancer specimens. Representative photos of non-metastasis and metastasis tumor versus the adjacent normal tissues were shown (magnification: $\times 100$ and $\times 400$ ). Scale bars: $100 \mu \mathrm{m}$. d MIIP pS303 levels in tumor and the adjacent normal tissues of colorectal cancer. e MIIP pS303 levels in tumor metastasis (M1) or non-metastasis subgroups (M0). The Chi-square test indicating the tight correlation between MIIP pS303 levels and tumor metastasis. $\mathbf{f}$ The survival times for 182 patients with low (score $<6$, blue curve) versus high ((score $\geq 6$, red curve) MIIP pS303 levels (low, 73 patients; high, 109 patients). The Kaplan-Meier method and log-rank tests indicating the significant association of MIIP pS303 levels ( $P=0.0235$ ) with patient survival. In $\mathbf{a}, \mathbf{d}$, ${ }^{\star}$ represents $P<0.05$ and ${ }^{\star *}$ represents $P<0.01$ (Student's $t$-test) between indicated groups

colorectal cancer cell metastasis, SW620 cells were reconstituted with expression of WT rMIIP, rMIIP S303A and rMIIP S303A/ HDAC6 shRNA (Supplementary Fig. 6a). EGF treatment in SW620 cells notably induced the interaction between WT MIIP and RelA but not MIIP S303A, and increased RelA Ac-K310 levels in cells with reconstituted expression of WT rMIIP instead of rMIIP S303A (Supplementary Fig. 6b). In addition, EGF notably promoted HDAC6-MIIP-RelA complex formation in a PKC $\varepsilon$ activation-dependent manner (Supplementary Fig. 6c). Then a liver metastasis mouse model was constructed via intraspleenic injection (Fig. 6a, left panel). Consequently, tumor cells expressed with WT MIIP exhibited the strong capability of metastasis. In contrast, expression of MIIP S303A dramatically inhibited liver metastasis (Fig. 6a, right panel). However, the impaired liver metastasis by MIIP S303A expression was significantly reversed by HDAC6 depletion (Fig. 6a, right panel). In accordance, immunoblotting analysis of mice tumor tissues indicated WT rMIIP but not rMIIP S303A underwent S303 phosphorylation during tumor growth, and RelA Ac-K310 levels was attenuated in tumor tissues from cells with rMIIP S303A expression in a HDAC6 dependent manner (Fig. 6b). Collectively, these results suggest MIIP-S303 phosphorylation maintains RelA Ac-K310 levels in colon cancer cells in vivo through its antagonistic effects on HDAC6, thus facilitates tumor metastasis.

To examine the clinical relevance of the PKCE-mediated MIIPS303 phosphorylation, we performed IHC-staining analyses in 182 serial sections of human colorectal cancer patient specimens (Fig. 6c and Supplementary Table 1). As a result, MIIP-S303 phosphorylation was increased in colorectal cancer cells compared with adjacent normal colorectal cells (Fig. 6c, d). Moreover, MIIP-S303 phosphorylation level was significantly correlated with colorectal cancer metastasis $(P=0.028$; Fig. 6c, e). Notably, 


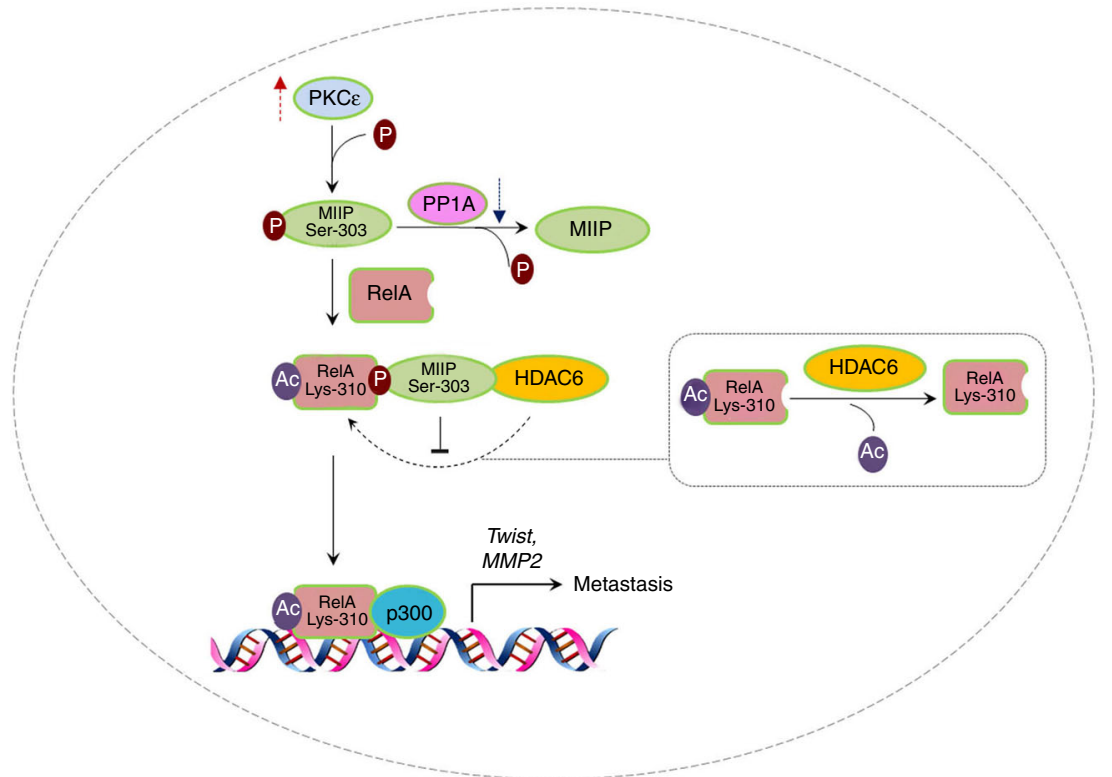

Fig. 7 A schematic diagram of model displaying the implication of MIIP in PKC $\varepsilon$ activation-induced tumor metastasis. PKC $\varepsilon$ phosphorylates MIIP at Ser303 and promotes its interaction with RelA, which protects RelA from deacetylation by HDAC6; in turn, this leads to enhancement of RelA transcriptional activity and increase of genes expression relevant to cell invasion. PP1 acts as a phosphotase being able to dephosphorylate MIIP phosphorylation by PKC $\varepsilon$. In cancer cells, PKC $\varepsilon$ activity is hyperactive while PP1 protein levels is downregulated; thereby, PKC $\varepsilon$ /MIIP/RelA signaling is reinforced and faciliates tumor metastasis

patients whose tumor expressed a high level (score $\geq 6$ ) of MIIPS303 phosphorylation had significantly shorter overall survival than those whose tumor expressed no or a low level (score $<6$ ) of MIIP-S303 phosphorylation (Fig. 6f). Furthermore, immunoblotting analysis of tumor tissues of clinical samples indicated MIIP S303 phosphorylation inversely correlates with PP1 levels (Supplementary Fig. 6d, e, upper panel); however, we found MIIP pS303 levels in tumor tissues were not correlated with total MIIP levels (Supplementary Fig. 6d, e, bottom panel), and MIIP levels even dropped in certain proportion of tumor tissues compared with the normal counterparts (Supplementary Fig. 6d). These results demonstrated the importantly indicative role of PKC $\varepsilon$-dependent MIIP-S303 phosphorylation in clinical behavior of human colorectal cancer.

\section{Discussion}

MIIP is identified as a suppressor in cell migration and invasion by its inhibitory effects on the relevant effectors such like IGFBP2 and HDAC6 ${ }^{13}$, yet how the functional status of MIIP is regulated remains unclear. In the present study, we show PKCe phosphorylates MIIP at Ser303 and promotes its binding to RelA; in turn, MIIP prevent RelA deacetylation from HDAC6 and facilitates RelA K310 acetylation-dependent expression of downstream genes including Twist, MMP2, thus promotes the cell capabilities of invasion (Fig. 7). These results illustrate a novel regulatory mechanism underlying the effect of MIIP on cell migration and invasion under the activation of EGF-PKC $\varepsilon$ signaling.

Expression levels of MIIP inversely correlate with tumor malignity ${ }^{17}, 26-29$. Consistent with previous reports ${ }^{26}$, we found interference of overall MIIP expression results in enhanced invasion basally in CRC cell, and MIIP levels are downregulated in tumor tissues of patients with stronger metastatic tendency. However, our study also shows MIIP depletion slightly suppresses the cell invasion instead under the condition of EGF activation (Fig. 1b); and replacement of WT MIIP with MIIP S303A mutant (the mutant resistant to $\mathrm{PKC} \varepsilon$ phosphorylation) dramatically inhibit EGF-induced cell invasion. Thus, these results indicate MIIP are switched into a driving force for cell invasion by $\mathrm{PKC} \varepsilon$ mediated phosphorylation, and this fraction might play a dominative role under EGF stimulation compared with the rest pool of MIIP that exerts negative effects on cell invasion. Furthermore, clinical analysis shows MIIP Ser303 phosophorylation levels positively correlate the poor prognosis in colorectal cancer patients, which suggests MIIP-regulated invasion in tumor cell is not only coupled with its expression level but also its phosophorylation status if EGF-PKC $\varepsilon$ signaling is taken into account. Of note, it does not mean our results contradict the established function of MIIP in concern of cell motility, as in previous studies the signaling context is not restricted ${ }^{16,17}$. In this regard, it can be assumed that MIIP Ser303 phosophorylation would become a decisive factor for malignity progression in tumors with wellexpressed MIIP.

PP1 is a serine/threonine specific protein phosphatase and deficiency of its function has been implicated in tumourigenesis of $\mathrm{CRC}^{19}, 30-32$. In our study, MIIP Ser303 phosphorylation is identified as a substrate of PP1. MIIP Ser303 phosphorylation displays an inverse relationship with PP1 protein levels in cancer cells. Therefore, MIIP Ser303 phosphorylation is also circumstantially regulated in a manner dependent on PP1 expression level, in addition to PKC $\varepsilon$ activity; given all this, it will be difficult to precisely evaluate MIIP function on cell invasion unless whole genetic background related is clearly defined.

MIIP is known to block cell migration through its inhibitory effects on the deacytalse activity of HDAC6 against alphatubulin $^{16}$. With respect to HDAC6, it is also able to inhibit cancer cell migration by removing RelA acetylation ${ }^{18}$. Thus the effect of HDAC6 on cell migration is context dependent. Our study shows MIIP with Ser303 phosphorylation potentiates cell invasion through its interaction with RelA, which can be explained by its concomitant binding to HDAC6 as the trimmer formed by RelA/ MIIP/HDAC6 is detected. Hence, MIIP negatively regulates HDAC6 in distinct signaling environmental conditions, while leads to contrary physiological outcome. Particularly, MIIP/RelA complex is exclusively distributed in the nucleus despite MIIP 
Ser303 phosphorylation can be detected in the cytosol; this reveals the function of MIIP with respect to cell migration and invasion is likely to be separated based on its subcellular localization; the access of cytosolic MIIP to RelA might be prevented by the abundant RelA-associated component in the cytosol.

Cancer invasion is associated with the abnormal regulation of components responsible for cell motility. The illustration of the mechanism by which MIIP inhibits RelA deacetylation under EGF activation sheds instrumental insight into how cell motility is subtly regulated in response to environmental stimulus; this finding importantly provides a molecular basis for developing therapy of malignant tumors with upregulated PKCE/MIIP/RelA signaling.

\section{Methods}

Cell culture. HCT116 cells were cultured in McCoy's 5A medium (Invitrogen) supplemented with $10 \%$ FBS. CaCo2 cells were cultured in minimum essential medium (MEM, Invitrogen) supplemented with 10\% FBS. SW1116, SW480 and SW620 cells were cultured in Leibovitz's L-15 medium (Invitrogen) supplemented with $10 \%$ FBS. All of the cell lines used in this study were obtained from ATCC and routinely tested for mycoplasma contamination. No cell lines used in this study were found in the database of commonly misidentified cell lines that is maintained by ICLAC and NCBI Biosample. The cell lines were not authenticated.

DNA constructs and mutagenesis. The pGIPZ human MIIP shRNA was generated with the oligonucleotides $5^{\prime}$-GCTACAACTCAGAGACTCC-3' for clone 1 , 5'-AGGAAGACCATGAATGCGT-3' for clone 2, and 5'-CTGAGTTG-

TAGCTGCTGCT-3' for clone 3. pGIPZ human PKC $\varepsilon$ was generated with the oligonucleotide 5'-CAAGTTCGGTATCCACAAC-3' and 5'-AGTTCATGGCCACCTATCT- $3^{\prime}$ shRNA pGIPZ human PP1 shRNA was generated with the oligonucleotide $5^{\prime}$-TCGTAGAAACCATAGATGC- $3^{\prime}$ and $5^{\prime}-$

CCAGGTTGAGCTTCTCGCT- ${ }^{\prime}$. pGIPZ human p300 shRNA was generated with the oligonucleotide 5'-CTAGAGACACCTTGTAGTA-3'. pGIPZ human HDAC6 shRNAs were generated with the oligonucleotides 5'-TTCAGGATGGGATGCAGTG-3'. The pGIPZ controls were generated with control oligonucleotide $5^{\prime}$-GCTTCTAACACCGGAGGTCTT- $3^{\prime}$ or $5^{\prime}$ GCCCGAAAGGGTTCCAGCTTA-3'

Materials. Antibodies that recognize PP1 (ab137512, 1:1000), PP2A (ab32141, 1:1000), Ac-RelAK310 (ab19870, 1:1000), Ac-H3K9 (ab10812, 1:1000), Ac-H3K18 (ab1191, 1:1000), H3K36Me2 (ab9049, H3K4Me3 (ab8580, 1:1000), and H3 (ab1791, 1:1000) were purchased from Abcam. Antibodies that recognize Flag (\#35535, 1:1000), Myc (\#T504, 1:1000), His (\#T505, 1:1000), an GST (\#T509, 1:1000) were obtained from Signalway Antibody. Antibodies against $\beta$-actin (\#4970, 1:2000), p-Acc (\#11818, 1:500), RelA (\#8242, 1:1000), and RelA pS536 (\#8214, 1:500) were purchased from Cell Signaling Technology. The antibody that recognize MIIP (HPA044948, 1:1000) were purchased from Sigma-Aldrich. Rabbit polyclonal MIIP pSer-303 antibody (1:500) was made by Signalway Antibody. A peptide containing MIIP pSer-303 antibody was injected into rabbits. The rabbit serum was collected and purified using an affinity column with nonphosphorylated MIIP Ser-303 peptide to exclude the antibodies for nonphosphorylated MIIP, followed by an affinity column with phosphorylated MIIP Ser-303 peptide to bind to and purify the MIIP pSer-303 antibody. The MIIP pSer303 antibody was then eluted and concentrated.

Bis-1 was purchased from Cell Signaling Technology (Boston, USA). EGF and U0126 were purchased from Sigma-Aldrich (St. Louis, MO). PKCE was from GeneTex (Irvine, USA). BrdU were purchased from Sigma-Aldrich (St. Louis, USA). OGT and OGA were ordered from R\&D (Minneapolis, USA). Nexturastat A, 4SC-202, and FK228 were from Selleckchem (Houston, USA).

Immunoprecipitation and immunoblotting analysis. Proteins were extracted from cultured cells using a modified buffer, followed by immunoprecipitation and immunoblotting with the corresponding antibodies. Proteins were extracted from cultured cells using a modified buffer (50 mM Tris- $\mathrm{HCl}$ ( $\mathrm{pH} 7.5), 1 \%$ Triton X-100, $150 \mathrm{mM} \mathrm{NaCl}, 0.5 \mathrm{mM}$ EDTA, $1 \mathrm{mM}$ dithiothreitol (DTT), and protease inhibitor cocktail or phosphatase inhibitor cocktail), followed by immunoprecipitation and immunoblotting with the corresponding antibodies. The protein concentration was determined through Bradford assay. Proteins from cellular lysates or nuclear extracts were separated by SDS-PAGE, transferred onto PVDF membrane (Millipore Corporation) and probed with the indicated antibodies. Unprocessed original scans of blots are shown in Supplementary Fig. 7.

Recombinant protein purification. WT and mutant GST-MIIP were expressed in bacteria and purified. Briefly, constructs were used to transform BL21/DE3 bacteria. The cultures were grown at $37^{\circ} \mathrm{C}$ to the OD $600 \mathrm{~nm}$ of $\sim 0.6$ before isopropyl$\beta$-D-thiogalactopyranoside treatment for $3 \mathrm{~h}$. Cell pellets were collected and lysed by sonication. For GST-tagged proteins, cleared lysates were bound to glutathioneagarose. For His-tagged proteins, cleared lysates were bound to Talon metal affinity resin. Eluates were concentrated using Ultrafree-15 centrifugal filters (Millipore).

Gene expression analysis. We isolated total RNA from cells using RNAzol RT (Molecular Research Center) following the manufacturer's instructions. We synthesized cDNA from $1 \mu \mathrm{g}$ total RNA using iScript cDNA synthesis kit (Bio-Rad) and quantified mRNA levels by real-time qRT-PCR using SYBR Green (Bio-Rad) We ran samples in technical triplicates and calculated relative mRNA levels normalized to actin mRNA levels in the same samples. The GPCR primer sequences were listed: human MMP2': 5'-CCACTGCCTTCGATACAC-3' (forward) and 5' GAGCCACTCTCTGGAATCTTAA-3' (reverse); human Twist: 5'-ACAAGCTGAGCAAGATTCAGACC-3' (forward) and 5'-TCCAGACCGAGAAGGCGTAG$3^{\prime}$ (reverse).

In vitro kinase assay. Purified WT and mutant GST-MIIP were incubated with PKC $\varepsilon$ in kinase assay buffer supplemented with $0.2 \mathrm{mM}$ AMP and cold ATP in the presence or absence of $0.2 \mathrm{mCi} / \mathrm{ml}$ hot ATP (ICN Biochemicals) for $20 \mathrm{~min}$ at $30^{\circ} \mathrm{C}$. After the reaction, PKC $\varepsilon$ was removed by extensive washing with RIPA buffer and kinase assay buffer. The GST-MIIP bound beads were recovered by centrifugation. For kinase phosphorylation analyses, the GST-MIIP bound beads were subjected to SDS-PAGE and then autoradiography after incubation with EN3HANCE (PerkinElmer). The GST-MIIP bound beads after PKCe treatment in absence of hot ATP were further incubate with cellular extracts as indicated.

ChIP assay. A ChIP assay was performed using an Upstate Biotechnology kit. Quantitative real-time PCR was used to measure the amount of bound DNA, and the value of enrichment was calculated according to the relative amount of input and the ratio to IgG. The primers covering RelA binding site of human Twistl gene promoter region were used for the real-time PCR: 5'-TTTGGGAGGACGAATTGTTAGACC-3' (forward) and 5'-TGGGCGAGAGCTGCAGACTTGG-3' (reverse); and the primers covering RelA binding site of human MMP2 gene promoter region were used for the real-time PCR: gene promoter region were used for the real-time PCR: 5'-CCACTGCCTTCGATACAC-3' (forward) and Reverse: 5'-GAGCCACTCTCTGGAATCTTAAA-3' (reverse).

Nuclear and cytoplasmic extracts. Cellular fractionation was performed using a Nuclear and Cytoplasmic Protein Extraction Kit (Beyotime Biotechnology, Shanghai, China). Briefly, the cells were harvested in ice-cold PBS and suspended in the hypotonic buffer with incubation for $15 \mathrm{~min}$ on ice. After vortex for $5 \mathrm{~s}$, the detergent was added into cells and incubated for 1 min on ice, followed by centrifugation at $16,000 \times g$ for $5 \mathrm{~min}$ at $4{ }^{\circ} \mathrm{C}$. The cytoplasmic fraction was transfered into separate tubes. The nuclear fraction was lysed in the complete lysis buffer on ice for $30 \mathrm{~min}$ and centrifuged at $16,000 \times \mathrm{g}$ for $10 \mathrm{~min}$ at $4{ }^{\circ} \mathrm{C}$.

Cell invasion assay. Cells were seeded in 24-well invasion chambers (BD Biosciences, San Jose, CA, USA) with the Matrigel-coated film insert ( $8 \mathrm{~mm}$ pore). The mixed solution was diluted to give a $1 \times$ DMEM solution containing $10 \%$ serum. The cells were seeded in absence or presence of EGF (100 ng/ml) (chemokinesis). Two days later, cells on the bottom surface of the filter subjected to staining with 4',6-diamidino-2-phenylindole for $1 \mathrm{~min}$, then washed three times with PBS, and the cell number was counted under a fluorescence microscope (Olympus).

Human tissue specimens and immunohistochemical analysis. Human tumor samples and their paired noncancerous matched tissues were obtained from 182 CRC patients treated at the hospital between 2008 and 2011. Written informed consent was obtained from each patient and the investigation was approved by the institutional review board of Zhongshan Hospital, Fudan University, Shanghai, China. Patients with radiotherapy or chemotherapy treatment before surgery were excluded. Survival time was calculated from the date of surgery to the date of death or last follow-up. Clinical information was collected from the medical records of each patient and shown in Supplementary Table 1. The tumor-node-metastasis (TNM) staging was performed according to American Joint Committee on Cancer (AJCC) standards.

Consecutive sections of formalin-fixed, paraffin-embedded (FFPE) tumors were subjected to IHC analysis for MIIP pS303. Rabbit polyclonal MIIP pSer-303 antibody (Signalway, 1:50) was used. A DAB substrate kit (GTVision Detection System/Mo\&Rb Kit) was used according to manufacturer's instructions. The results were scored by two pathologists blinded to the clinicopathologic data.

Mouse. All animal experiments were approved by the animal care and use committee of Fudan University. Twenty-four female Balb/c nude mice (5-week-old) were divided into three groups (eight mice per group): SW620 cells were reconstituted with expression of WT rMIIP, rMIIP S303A, and rMIIP S303A/ HDAC6 shRNA. A small left abdominal flank incision was made, the spleen was exteriorized, and the prepared cells $\left(2 \times 10^{6}\right.$ cells $/ 50 \mu \mathrm{l} /$ mouse $)$ were injected into the spleen with a 30 -Gauge needle. To prevent tumor cell leakage and bleeding, a cotton swab was held over the site of injection for $1 \mathrm{~min}$. The injected spleen was 
returned to the abdomen and the wound was sutured with 6-0 black silk. Six weeks later, all of the mice were sacrificed and necropsied for observation of visible metastatic lesions in the liver.

Statistical analysis. Differences between groups were calculated using the Student $t$-test, chi-square test, or the Fisher exact test. The log-rank test was used to obtain a $P$-value for the significance of divergence of Kaplan-Meier curves. All probability values were two-sided. Analyses were performed with the SPSS version 22.0 and Graphpad Prism 6.02 statistical analysis software. Statistical significance was defined as $P<0.05$.

Data availability. All relevant data supporting the findings of this study are available from the corresponding author on reasonable request.

Received: 11 December 2016 Accepted: 14 August 2017

Published online: 16 October 2017

\section{References}

1. Karin, M. \& Delhase, M. The I kappa B kinase (IKK) and NF-kappa B: key elements of proinflammatory signalling. Semin. Immunol. 12, 85-98 (2000).

2. Luo, J. L., Kamata, H. \& Karin, M. The anti-death machinery in IKK/NFkappaB signaling. J. Clin. Immunol. 25, 541-550 (2000).

3. Karin, M. Nuclear factor-kappaB in cancer development and progression. Nature 441, 431-436 (2006)

4. Luo, J. L., Kamata, H. \& Karin, M. IKK/NF-kappaB signaling: balancing life and death--a new approach to cancer therapy. J. Clin. Invest. 115, 2625-2632 (2005).

5. Karin, M. \& Greten, F. R. NF-kappaB: linking inflammation and immunity to cancer development and progression. Nat. Rev. Immunol. 5, 749-759 (2005).

6. Karin, M. How NF-kappaB is activated: the role of the IkappaB kinase (IKK) complex. Oncogene 18, 6867-6874 (1999).

7. Ghosh, S. \& Hayden, M. S. New regulators of NF-kappaB in inflammation. Nat. Rev. Immunol. 8, 837-848 (2008).

8. Shostak, K. \& Chariot, A. EGFR and NF-kappaB: partners in cancer. Trends Mol. Med. 21, 385-393 (2015).

9. Garg, R. et al. Activation of nuclear factor kappaB (NF-kappaB) in prostate cancer is mediated by protein kinase $\mathrm{C}$ epsilon (PKCepsilon). J. Biol. Chem. 287, 37570-37582 (2012).

10. Yang, W. et al. EGFR-induced and PKCepsilon monoubiquitylation-dependent NF-kappaB activation upregulates PKM2 expression and promotes tumourigenesis. Mol. Cell 48, 771-784 (2012).

11. Perkins, N. D. Post-translational modifications regulating the activity and function of the nuclear factor kappa B pathway. Oncogene 25, 6717-6730 (2016).

12. Greene, W. C. \& Chen, L. F. Regulation of NF-kappaB action by reversible acetylation. Novartis Found. Symp. 259, 208-217 (2004). discussion 218-225.

13. Ji, P. et al. Inhibition of gliomagenesis and attenuation of mitotic transition by MIIP. Oncogene 29, 3501-3508 (2010)

14. Wang, Y., Wen, J. \& Zhang, W. MIIP, a cytoskeleton regulator that blocks cell migration and invasion, delays mitosis, and suppresses tumourogenesis. Curr. Protein Pept. Sci. 12, 68-73 (2011).

15. Song, S. W. et al. IIp45, an insulin-like growth factor binding protein 2 (IGFBP2) binding protein, antagonizes IGFBP-2 stimulation of glioma cell invasion. Proc. Natl Acad. Sci. USA 100, 13970-13975 (2003).

16. Wu, Y. et al. IIp45 inhibits cell migration through inhibition of HDAC6. J. Biol. Chem. 285, 3554-3560 (2010)

17. Wen, J., Fu, J., Ling, Y. \& Zhang, W. MIIP accelerates epidermal growth factor receptor protein turnover and attenuates proliferation in non-small cell lung cancer. Oncotarget 7, 9118-9134 (2016)

18. Yang, C. J. et al. Nuclear HDAC6 inhibits invasion by suppressing NF-kappaB/ MMP2 and is inversely correlated with metastasis of non-small cell lung cancer. Oncotarget 6, 30263-30276 (2015).

19. Cohen, P. T. Protein phosphatase 1--targeted in many directions. J. Cell Sci. 115, 241-256 (2002).

20. Ahmed, D. et al. Epigenetic and genetic features of 24 colon cancer cell lines. Oncogenesis 2, e71 (2013).

21. Hoberg, J. E., Popko, A. E., Ramsey, C. S. \& Mayo, M. W. IkappaB kinase alphamediated derepression of SMRT potentiates acetylation of RelA/p65 by p300. Mol. Cell. Biol. 26, 457-p471 (2006).
22. Allison, D. F. et al. Modification of RelA by O-linked N-acetylglucosamine links glucose metabolism to NF-kappaB acetylation and transcription. Proc. Natl Acad. Sci. USA 109, 16888-16893 (2012).

23. Kawahara, T. L. et al. SIRT6 links histone H3 lysine 9 deacetylation to NFkappaB-dependent gene expression and organismal life span. Cell 136, 62-74 (2009).

24. Roth, S. Y., Denu, J. M. \& Allis, C. D. Histone acetyltransferases. Annu. Rev. Biochem. 70, 81-120 (2001).

25. Ogryzko, V. V., Schiltz, R. L., Russanova, V., Howard, B. H. \& Nakatani, Y. The transcriptional coactivators p300 and CBP are histone acetyltransferases. Cell 87, 953-959 (1996).

26. Sun, Y. et al. MIIP haploinsufficiency induces chromosomal instability and promotes tumour progression in colorectal cancer. J. Pathol. 241, 67-79 2016

27. Wen, J. et al. MIIP expression predicts outcomes of surgically resected esophageal squamous cell carcinomas. Tumour Biol. 37, 10141-10148 (2016).

28. Song, F. et al. Definition of a functional single nucleotide polymorphism in the cell migration inhibitory gene MIIP that affects the risk of breast cancer. Cancer Res. 70, 1024-1032 (2010).

29. Wang, Y. et al. MIIP remodels Racl-mediated cytoskeleton structure in suppression of endometrial cancer metastasis. J. Hematol. Oncol. 9, 112 (2016)

30. Dickinson, R. J. \& Keyse, S. M. Diverse physiological functions for dualspecificity MAP kinase phosphatases. J. Cell Sci. 119, 4607-4615 (2006).

31. Eke, I. et al. PINCH1 regulates Aktl activation and enhances radioresistance by inhibiting PP1alpha. J. Clin. Invest. 120, 2516-2527 (2010).

32. Castro, M. E. et al. PPP1CA contributes to the senescence program induced by oncogenic Ras. Carcinogenesis 29, 491-499 (2008).

\section{Acknowledgements}

This work was supported by the National Key R\&D Program of China 2017YFA0506200 (Y.J.), National Nature Science Foundation of China 81572722 (Y.J.), National Nature Science Foundation of China 81502523 (T.C.), Shanghai Committee of Science and Technology 16QA1403200 (Y.J.), Shanghai Municipal Education Commission-Gaofeng Clinical Medicine Grant 20161319 (Y.J.), Program for eastern young scholar at Shangha institutions of higher learning (Y.J.), Shanghai Committee of Science and Technology 16DZ2280990 (L.Y.)

\section{Author contributions}

This study was conceived by Y.J.; Y.J., T.C. and P.Z. designed the study; T.C. and J.L. performed the experiments; Y.J., T.C., J.L., Q.Z., M.X., L.Y., Y.Z. and Y.H. analyzed and interpreted the data; T.C. and Y.J. wrote and revised the manuscript. W.Z. and P.C. reviewed the manuscript and gave conceptual advice; Y.J., P.Z. and W.Z. technical and material support. All authors approved the manuscript.

\section{Additional information}

Supplementary Information accompanies this paper at doi:10.1038/s41467-017-01024-2.

Competing interests: The authors declare no competing financial interests.

Reprints and permission information is available online at http://npg.nature.com/ reprintsandpermissions/

Publisher's note: Springer Nature remains neutral with regard to jurisdictional claims in published maps and institutional affiliations.



Open Access This article is licensed under a Creative Commons Attribution 4.0 International License, which permits use, sharing, adaptation, distribution and reproduction in any medium or format, as long as you give appropriate credit to the original author(s) and the source, provide a link to the Creative Commons license, and indicate if changes were made. The images or other third party material in this article are included in the article's Creative Commons license, unless indicated otherwise in a credit line to the material. If material is not included in the article's Creative Commons license and your intended use is not permitted by statutory regulation or exceeds the permitted use, you will need to obtain permission directly from the copyright holder. To view a copy of this license, visit http://creativecommons.org/ licenses/by/4.0/

(c) The Author(s) 2017 